\title{
Mmu-miR-27a-5p Promotes the Polarization of Macrophages to the M2 Phenotype at the Site of Spinal Cord Injury in Mice
}

\section{Mingkun Yang}

First affiliated hospital of Xian Jiaotong University

Xiaoqian Dang ( $\nabla$ dangxiaoqian1122@163.com)

Second affiliated hospital of Xi'an Jiaotong university

\section{Xu Zhang}

Bazhong Central hospital

Chuan Liu

Bazhong center hospital

Min $\mathrm{He}$

Bazhong center hospital

\section{Research}

Keywords: mmu-miR-27a-5p, spinal cord injury, macrophages polarization, M2 phenotype

Posted Date: January 5th, 2021

DOI: https://doi.org/10.21203/rs.3.rs-111721/v1

License: (c) (1) This work is licensed under a Creative Commons Attribution 4.0 International License. Read Full License 


\section{Abstract}

\section{Background}

To investigate the effect of mmu-miR-27a-5p on macrophage polarization in the injured spinal cord and the recovery of motor function after spinal cord injury $(\mathrm{SCl})$ in mice.

\section{Methods}

A total of 160 specific-pathogen-free male mice were randomly divided into sham, model, mmu-miR-27a$5 p$, mmu-miR-27a-5p-negative control (NC) groups, with 40 mice in each group. Hindlimb motor function was assessed using the Basso Mouse scale (BMS) before injury and at 1, 3, 7, and 14 days after surgery. Spinal cord tissue samples were obtained at 1, 3, 7, and 14 days after surgery, and macrophage polarization types were detected by using western blot analysis, immunofluorescence, flow cytometry and RT-qPCR.

\section{Results}

The BMS score in the mmu-miR-27a-5p group was significantly higher than that in the model and mmumiR-27a-5p-NC groups at 7 and 14 days after $\mathrm{SCl}(X 2=26.45-57.62, \mathrm{P}<0.05)$. No significant changes in the expression of M1 markers IL-1 $\beta$, TNF- $\alpha$ and M2 markers IL-10, Arginase-1 at each time point in the sham group ( $\mathrm{P}=0.96)$. The expression of IL-1 $\beta$ and TNF-a was significantly lower, while the expression of IL-10 and Arginase-1 were significantly higher in the mmu-miR-27a-5p group as compared to the model and mmu-miR-27a-5p-NC groups at 7 and 14 days after $\mathrm{SCl}(\mathrm{P}<0.05)$.

\section{Conclusion}

Administration of mmu-miR-27a-5p can promote the polarization of macrophages to the M2 phenotype in the injured spinal cord, and improve motor function recovery within 14 days after $\mathrm{SCl}$ in mice.

\section{Background}

Spinal cord injury ( $\mathrm{SCl}$ ) is a serious medical condition, and there is currently no effective treatment for $\mathrm{SCl}$, which brings heavy burden to families and society [1]. SCl is accompanied by a series of inflammatory responses. At the injury site, microglia and infiltrating monocytes are activated and differentiated into macrophages from 1 day to several weeks after injury. Accoring to macrophage polarization states, macrophages can be divided into M1and M2 phenotypes. MI macrophages can promote inflammation, engulf local necrotic cells and cell debris, leading to increased glial scarring, increased empty cavities, axonal retraction, and exacerbated neurological impairment. M2 macrophages can inhibit inflammation, protect cells, and secrete nerve growth factors to promote tissue repair [2]. Macrophage polarization is affected by many factors, mmu-miR-27a-5p plays an important role in this process, and the mechanism may be related to its regulatory effect on MCPIP1 expression [3]. At present, the effect of mmu-miR-27a-5p on macrophage polarization after SCl has not yet been studied. This study 
aimed to explore the effect of mmu-miR-27a-5p on the polarization of macrophage in the injured spinal cord in mouse model of SCl.

\section{Results}

\section{Behavioral scoring results}

There was no statistically significant difference in BMS score between the four groups before $\mathrm{SCl}(\mathrm{P}=$ 1.0). The hindlimb function disappeared within 1 day after $\mathrm{SCl}(\mathrm{BMS}$ score $=0)$, and gradually recovered thereafter in both model, mmu-miR-27a-5p, and mmu-miR-27a-5p-NC groups. BMS score returned to the preoperative level on day 3 after surgery in the sham group. The BMS scorewas significantly higher in the mmu-miR-27a-5p group than in the model and mmu-miR-27a-5p-NC groups at 7 and 14 days after $\mathrm{SCl}$ $(X 2=26.45-57.62, P<0.05)$, indicating that the mice in the mmu-miR-27a-5p group showed better recovery of hindlimb and trunk motor function.

\section{Expression of macrophage markers detected by western blot analysis}

No significant changes were observed in the expression of IL-1 $\beta$, TNF-a, IL-10, and Arginase-1 at each time point in the sham group $(P=0.96)$. There was no statistically significant difference in the expression of IL-1 $\beta$, TNF- $a$, IL-10, and Arginase- 1 between mmu-miR-27a-5p, model and mmu-miR-27a-5p-NC groups at 1 and 3 days after $S C I(P=0.84)$. The expression of IL-1 $\beta$ and TNF- $\alpha$ was significantly lower in the mmu-miR-27a-5p group as compared to model and mmu-miR-27a-5p-NC groups at 7 and 14 days after $\mathrm{SCl}$. On the contrary, the expression of IL-10 and Arginase- 1 were significantly higher in the mmu-miR-27a$5 p$ group as compared to the model and mmu-miR-27a-5p-NC groups at 7 and 14 days after $S C(P<0.05$, Fig. 2). It is suggested that there were more M2 macrophages in the injury site of mice in the MMU-miR27a-5p group.

\section{Immunofluorescent staining results for macrophage markers}

No significant change was observed in the expression of IL-1 $\beta$, TNF- $a$, IL-10, and Arginase- 1 at each time point after $\mathrm{SCl}$ in the sham group. There were also no significant difference in the expression of IL-1 $\beta$, TNF-a, IL-10, and Arginase- 1 at 1 and 3 days after SCl between the mmu-miR-27a-5p, model and mmumiR-27a-5p-NC groups. At 7 and 14 days after $\mathrm{SCl}$, there were significantly increased numbers and wider distributions of IL-10- and Arginase-I -positive cells, and significantly decreased numbers and restricted distributions of IL-1 $\beta$ - and TNF-a-positive cells in the mmu-miR-27a-5p group compared to the model and mmu-miR-27a-5p-NC groups (Fig. 2), indicating that mice in the mmu-miR-27a-5p group had more M2 macrophages in the injured spinal cord. 


\section{Flow cytometry results for detection of macrophage markers}

Flow cytometry was used to detect M1 marker, CD16/32, and M2 marker, Arginase-1. At 7 and 14 days after SCl, M1 marker CD16/32 was significantly declined in the mmu-miR-27a-5p group compared with the model and mmu-miR-27a-5p-NC groups $(P=0.000)$. On the contrary, M2 marker Arg- 1 was significantly increased in the mmu-miR-27a-5p group compared with the model and mmu-miR-27a-5p-NC groups $(P=0.000$, Fig. 3$)$. The results indicated that mmu-miR-27a-5p can promote the polarization of macrophages to M2 phenotype in the injured spinal cord.

\section{Expression of macrophage markers detected by RT-PCR}

No significant change was observed in the relative expression of each macrophage marker gene in the sham group $(P>0.05)$. Relative expression of IL-10 and Arginase- 1 were significantly increased, while relative expression of IL-1 $\beta$ and TNF- $\alpha$ were significantly decreased at 7 and 14 days after $S C l$ in the mmu-miR-27a-5p group than those in the model and mmu-miR-27a-5p-NC groups $(P<0.05)$, indicating that administration of mmu-miR-27a-5p can inhibit the inflammatory responses, affect macrophage polarization types in the injury site by decreasing M1 macrophage polarization and increasing M2 macrophage polarization within 14 days following SCl.

\section{Discussion}

Inflammatory response mainly affects axonal regeneration and functional recovery after SCl. Changes in local microenvironment occur rapidly after $\mathrm{SCl}$, neutrophil infiltration and microglia activation are first initiated, followed by accumulation of monocyte-derived macrophages [6, 7], macrophages recognize and scavenge substances at the injury site that are not conducive to nerve repair and promote tissue regeneration [8], reduce or prevent the expanded secondary injury. In this study, we found that changes in BMS scores for hindlimb motor function were basically consistent with the changes in the expression of IL-1 $\beta$ and TNF- $\alpha$ at 7 days after injury, as well as the change in the expression levels of Arginase- 1 and IL10 at $14 \mathrm{~d}$ after $\mathrm{SCl}$, suggesting that macrophages play a positive role in the recovery of spinal cord function after SCl. A study [7] established a mouse model of traumatic brain injury and found that macrophages isolated from the ipsilateral cerebral cortex were activated due to the expression of NADPH oxidase 2 at 7 days post injuy, which can significantly inhibit neuronal growth and enhance inflammatory responses. The reuslts indicated that after central nervous system injury, the macrophage-mediated inflammatory response can also hinder neural repair. Therefore, macrophages play a dual role in $\mathrm{SCl}$ repair. Findings from our study and the above mentioned previous studies both suggest that the mechanism may be related to different polarization types of macrophages caused by changes in the local microenvironment after SCl.

Due to different stimulating factors and difference in the microenvironment at the injury sites, macrophages can be polarized into M1 and M2 types, which secrete different cytokines and chemokines, 
and exert different effects $[9,10]$. mmu-miR-27a-5p plays an important role in macrophage polarization, which can affect the polarization of macrophages by regulating MCPIP1 expression [3]. In this study, highest expression of M1 markers IL-1 $\beta$ and TNF-a was observed at 7 days after $\mathrm{SCl}$, while highest expression of M2 markers Arginase-1, IL-10 was observed at 14 days after $\mathrm{SCl}$ in the model and mmumiR-27a-5p-NC groups. IL-1 $\beta$ and TNF-a expression levels were significantly lower, and Arginase-1 and IL10 expression levels were significantly higher at 7 and 14 days after $\mathrm{SCl}$ in the mmu-miR-27a-5p group compared with the model and mmu-miR-27a-5p-NC groups. These results indicate that administration of mmu-miR-27a-5p can increase M2 macrophage polarization at the injury sites, which have beneficial effects on the repair of injured spinal cord. Fan et al. [11] believed that after SCl, M1 macrophages induce the apoptosis of reactive astrocytes through the TLR/MyD88 signaling pathway and promote glial scar formation. M2 macrophages can promote the growth of neuronal axons [12], the underlying mechanism may be that M2 macrophages secrete large amounts of fibrous protein and matrix-related proteins, inhibit macrophage infiltration, the expression of inflammatory cytokines and activation of their receptors following $\mathrm{SCl}$, alleviate oxidative stress and secondary $\mathrm{SCl}$, and promote endothelial cell proliferation, angiogenesis, and neural function recovery after $\mathrm{SCl}$ [13-15]. Our results also confirmed that at $7 \mathrm{~d}$ and $14 \mathrm{~d}$ following $\mathrm{SCl}, \mathrm{BMS}$ scores in the mmu-miR-27a-5p group was significantly higher than that in the model and mmu-miR-27a-5p-NC groups $(X 2=26.45-57.62, P<0.05)$. Administration of mmu-miR-27a-5p can promote recovery of hindlimb function in mice after $\mathrm{SCl}$.

In fact, the polarization types of macrophages is far more complex than characterized by the M1/M2 polarization. M1 and M2 macrophages may represent two extreme ends of macrophage polarization, there are intermediate phenotypes. At present, three subtypes of M2 macrophages have been distinguished: $\mathrm{M} 2 \mathrm{a}, \mathrm{M} 2 \mathrm{~b}$, and $\mathrm{M} 2 \mathrm{c}$, each one has its own characteristics and functions. After SCl, M1 and $\mathrm{M} 2 \mathrm{a}$ macrophages appeared at the early stage after $\mathrm{SCl}$, followed by M2b and M2c macrophages, and finally overall decrease in M2 macrophage can be observed. M1 gradually occupied and maintained a dominant position for a long time at 1 week following $\mathrm{SCl}$ [16-18], this is one of the reason why patient with $\mathrm{SCl}$ can not recover in a long time. In this study, we detected the protein and mRNA expression of M1 phenotypic markers (IL-1 $\beta$ and TNF- $a$ ), and M2a phenotypic markers (arginase-1 and IL-10), and we observed decreased expression of M1 phenotypic markers, and increased expression of M2a phenotypic markers in the mmu-miR-27a-5p group compared with the mmu-miR-27a-5p-NC group, we conclude that administration of mmu-miR-27a-5p can increase M2a macrophage and decrease M1 macrophage. Since the total number of macrophages is constant, the increase in M2a macrophage at early stage (within $3 \mathrm{~d}$ ) following $\mathrm{SCl}$ may be accompanied by a decrease in other macrophage subtypes. The trends of changes in M2b and M2c macrophages need to be further investigated using more specific phenotypic markers.

The limitation of this study is that we mainly focused on the changes in the polarization types of macrophages at the site of injury, did not observe the changes in the macrophages derive from blood monocytes, further study are needed to confirm our findings.

In summary, administration of mmu-miR-27a-5p can promote the polarization of macrophages to M2 phenotype in the site of injury, improve the recovery of motor function in mice following SCl. 


\section{Materials And Methods \\ Experimental animals and grouping}

A total of 160 specific-pathogen-free C57BL/6j male mice (45-56 days old) were purchased from Three Gorges University. Mice were randomly divided into sham, model, mmu-miR-27a-5p, mmu-miR-27a-5pnegative control (NC) groups, with 40 mice in each group. The animal study was approved by the ethics committee of our hospital.

\section{Preparation of mmu-miR-27a-5p mimics}

mmu-miR-27a-5p mimics was synthesized by Wuhan Biofavor Biotech Services Co., Ltd. (Wuhan Biofavor Biotech Services Co., Ltd., Wuhan, China), an unrelated universal sequence was as a negative control (NC). RAW 264.7 macrophages were seeded into 6-well plates and cultured with mmu-miR-27a-5p mimics and mmu-miR-27a-5p-NC, respectively, at $37^{\circ} \mathrm{C}$ overnight. Lentivirus vector solution $(1 \times 109 \mathrm{TU} / \mathrm{ml})$ were mixed and diluted with medium at a multiplicity of infection of $100,10,1$. The old medium was aspirated from the 6-well plate, and replaced with the virus gradient dilutions. After $24 \mathrm{~h}$ of culture at $37^{\circ} \mathrm{C}$, culture medium was replaced with fresh media. After 48 hours of culture, transfection efficiency was observed under a fluorescent microscope. The optimal transfection condition was chosen and used for subsequent experiments.

\section{SCl modeling}

After 3 days of acclimation, all mice were anesthetized intraperitoneally with $4 \%$ chloral hydrate. After hair removal and skin disinfection in the surgical field, a longitudinal incision was made centered on T10 spinous process, then the T9, T10 spinous processes and lamina were removed to expose the dura mater. In the model, mmu-miR-27a-5p, and mmu-miR-27a-5p-NC groups, SCl at T9-10 levels was produced by freely dropping a $10 \mathrm{~g}$ weight from a height of $5 \mathrm{~cm}$ using a weight drop device onto the T9-10 spinal segment [17]. The signs of successful model establishment included spastic swinging of the tail and retraction-like flutter and flaccid paralysis of the hind limbs immediately after injury. In the sham group, T9-10 spinous processes and lamina were removed without damaging the spinal cord. After surgery, the mice were kept warm. And after recovery from anaesthesia, mice were moved to the animal room. All mice were fed normally. 20,000 units of penicillin were injected intraperitoneally into mice once a day within 3 days after surgery, and the bladder of the mice was squeezed manually twice daily. If mice died during surgery, new mice was added and animal model was established again.

\section{Treatment}

All mice in each group were treated at day 3 after $\mathrm{SCl}$ model was successfully established. Mice in sham group received no treatment, mice in model group were treated with $10 \mu \mathrm{l}$ saline. Mice in mmu-miR-27a-5p group and mmu-miR-27a-5p-NC group received $10 \mu \mathrm{l}$ of mmu-miR-27a-5p suspension and mmu-miR-27a$5 p-N C$ suspension, respectively. Before administration, mmu-miR-27a-5p suspension and mmu-miR-27a$5 p-N C$ suspension were co-cultured with $\mathrm{BrdU}(10 \mu \mathrm{mol} / \mathrm{L})$ for 72 hours. Multi-point injections were slowly 
given for 5 minutes at the injured spinal cord, the needle was left in place for additional 5 min before removal, then feeding was continued.

\section{Behavioral testing}

Before surgery and at $1,3,7$, and 14 days after surgery, open field test was perfromed [5], mice were placed in an open field, allowed to freely explore fo for $5 \mathrm{~min}$. Hindlimb and trunk motor function were assessed using Basso Mouse scale (BMS) by a examiner who did not participate in the study design.

\section{Spinal cord tissue acquisition}

After successful established of SCI model, 10 mice of each group were sacrificed at 1, 3, 7, and 14 days after SCl to collect their spinal cord samples. Mice were deeply anesthetized with ketamine/xylazine, and perfused through the heart with saline, followed by $40 \mathrm{~g} / \mathrm{L}$ paraformaldehyde. Approximately $1.5 \mathrm{~cm}$ length of spinal cord tissue centered at the injury site was taken, placed in paraformaldehyde overnight at $4{ }^{\circ} \mathrm{C}$, dehydrated in $30 \%$ sucrose solution at $4{ }^{\circ} \mathrm{C}$ until sinking. Then the tissue was embedded in an optimal cutting temperature, $20-\mu \mathrm{m}$ sections were cut, and frozen at $-80^{\circ} \mathrm{C}$.

\section{Detection of macrophage markers using western blot analysis}

The collected spinal cord tissues were homogenized in RIPA lysis buffer (Beyotime Biotechnology, China) at $4{ }^{\circ} \mathrm{C}$ for 5 min to obtain $10 \%$ homogenate. The homogenates were lysed in RIPA buffer at $4{ }^{\circ} \mathrm{C}$ for $45 \mathrm{~min}$, centrifuged at $12000 \mathrm{r} / \mathrm{min}$ for $5 \mathrm{~min}$, then the supernatant were collected. The BCA protein assay kit was used to measure total protein concentration according to the manufacturer's protocol. Protein samples were separated on SDS-PAGE gels and transferred to PVDF membranes. The membranes were blocked in $5 \%$ non-fat dry milk in TBS and Tween-20 for $1.5 \mathrm{~h}$, incubated with primary antibodies against M1 markers IL-1 $\beta$, TNF- $a$ and M2 markers IL-10, Arginase-1. Immunoblots were visualized using the ECL western blotting detection system

\section{Detection of macrophage markers using immunofluorescence staining}

Sections were permeabilized and blocked in $0.2 \%$ Triton X-100 in PBS, and incubated with primary antibodies against Arginase-1 (1:100, WACO, Osaka, Japan), IL-10 (1:200, WACO, Osaka, Japan), TNF-a (1:30, WACO, Osaka, Japan), IL-1 $\beta$ (1:200, WACO, Osaka, Japan) overnight at $4{ }^{\circ} \mathrm{C}$. After the sections were rinsed, the sections were incubated for $2 \mathrm{~h}$ at room temperature with species-specific secondary antibodies, including CY3 Goat Anti-Rabbit IgG Antibody (1:100 dilution), Alexa-594 (red)-conjugated TNFaand IL-1ßantibodies (1:250, Invitrogen, USA), Alexa Fiuor-488 (green)-conjugated Arginase-1, IL-10 antibodies (1:250, Invitrogen, USA). Finally, sections were washed, mounted with DAPI, and visualized using fluorescent microscope (DM3000) or a confocal laser scanning microscope (TCS SP5 II; both Leica Microsystems, Inc., Germany). Three non-overlapping fields at the injury site were randomly selected in 
each slice at 400 magnification. Positively stained cells were counted from the nine randomly selected microscopic fields by using the Image-Pro Plus (IPP 6.0) software and the mean values were calculated.

\section{Detection of macrophage markers using flow cytometry}

$1 \mathrm{ml}$ of PBS containing $0.5 \%$ bovine serum albumin and $1 \times 10^{6}$ cells were added to each flow tube, and incubated with PE-conjugated CD16/CD32 monoclonal antibody (clone 93, $0.125 \mu \mathrm{g}$ per test, BD Biosciences, USA), and APC-conjugated Arginase- 1 monoclonal antibody (A1 exF5, $1.0 \mu \mathrm{g}$ per test) at $4{ }^{\circ} \mathrm{C}$ in the dark for $30 \mathrm{~min}$. After centrifugation at $15000 \mathrm{r} / \mathrm{mim}$ for $5 \mathrm{~min}$, the supernatant was discarded, then cells were washed once with $1 \mathrm{ml} \mathrm{PBS}$, and centrifuged at $15000 \mathrm{r} / \mathrm{mim}$ for $3 \mathrm{~min}$. The supernatant was removed and the cells were resuspended in $0.2 \mathrm{ml}$ PBS containing $0.5 \%$ BSA. Data were analyzed using flow cytometer (Attune NxT, Thermo Fisher Scientific, Inc. USA).

\section{Detection of macrophage markers using real-time quantitative PCR (RT-qPCR)}

Total RNA was extracted using the Trizol reagent (Thermo Fisher Scientific, Inc. USA). Reverse transcription for DNA was synthesized using PrimeScript ${ }^{\mathrm{TM}}$ reverse transcription kit (Takara, Japan). RTqPCR was performed in a $10 \mu \mathrm{L}$ reaction system using UltraSYBR premixture (CWbio Co., Ltd., Beijing, China). RT-qPCR conditions included $10 \mathrm{~min}$ of predenaturation at $95^{\circ} \mathrm{C}$, followed by 35 cycles of denaturation at $95^{\circ} \mathrm{C}$ for $15 \mathrm{~s}$, annealing/ elongation at $60^{\circ} \mathrm{C}$ for $1 \mathrm{~min}$. The dissolution curve conditions were $95^{\circ} \mathrm{C}$ for $15 \mathrm{~S}, 60^{\circ} \mathrm{C}$ for $1 \mathrm{~min}, 95^{\circ} \mathrm{C}$ for $15 \mathrm{~s}, 60^{\circ} \mathrm{C}$ for $15 \mathrm{~s}$. The relative quantification was calculated using the ${ }^{2-\Delta \Delta C T}$ method with reference genes $\beta$-actin.

\section{Statistical analysis}

Statistical analysis was performed using GraphPad Prism 6.01 software. Measurement data were expressed as mean \pm standard deviation (mean $\pm S D$ ). Differences in the results from RT-PCR and immunofluorescence staining were analyzed using $t$ test, and differences in the results of behavioral scoring were analyzed using repeated measures Analysis of Variance (ANOVA). A difference with a Pvalue of $<0.05$ was considered statistically significant.

\section{Abbreviations}

SCl,spinal cord injury

NC group, control group

BMS, Basso Mouse scale

RT-qPCR, real-time quantitative PCR

\section{Declarations}

Acknowledgements 
Not applicable.

\section{Funding}

This work was supported by the science and technology project of Sichuan province (2018JY0308).

\section{Availability of data and materials}

All data generated or analyzed during this study are included in this published article.

\section{Authors' contributions}

XD conceived and designed the experiments; MK wrote the paper; MK, XD performed the experiments; XZ, $\mathrm{CL}, \mathrm{MH}$ analyzed the data and contributed reagents/materials/analysis tools.

\section{Availability of data and materials}

All data generated or analyzed during this study are included in this published article.

\section{Ethics approval and consent to participate}

The animal study was approved by the ethics committee of Second Affiliated Hospital of Xi'an Jiaotong University.

\section{Consent for publication}

All authors read the final manuscript and approved for publication.

\section{Competing interests}

The authors declare that they have no competing interests.

\section{References}

1. Silva NA, Sousa N, Reis RL, Salgado AJ. From basics to clinical: a comprehensive review on spinal cord injury. Prog Neurobiol. 2014 ;114:25-57. doi: 10.1016/j.pneurobio.2013.11.002.

2. Haan N, Zhu B, Wang J, Wei X, Song B. Crosstalk between macrophages and astrocytes affects proliferation, reactive phenotype and inflammatory response, suggesting a role during reactive gliosis following spinal cord injury. J Neuroinflammation. 2015;12:109. doi: 10.1186/s12974-015-0327-3.

3. Cheng Y, Du L, Jiao H, Zhu H, Xu K, Guo S, Shi Q, Zhao T, Pang F, Jia X, Wang F. Mmu-miR-27a-5pDependent Upregulation of MCPIP1 Inhibits the Inflammatory Response in LPS-Induced RAW264.7 Macrophage Cells. Biomed Res Int. 2015;2015:607692. doi: 10.1155/2015/607692.

4. Chen WF, Chen CH, Chen NF, Sung CS, Wen ZH. Neuroprotective Effects of Direct Intrathecal Administration of Granulocyte Colony-Stimulating Factor in Rats with Spinal Cord Injury. CNS 
Neurosci Ther. 2015;21(9):698-707. doi: 10.1111/cns.12429.

5. Basso DM, Fisher LC, Anderson AJ, Jakeman LB, McTigue DM, Popovich PG. Basso Mouse Scale for locomotion detects differences in recovery after spinal cord injury in five common mouse strains. $J$ Neurotrauma. 2006;23(5):635-59. doi: 10.1089/neu.2006.23.635.

6. Shechter R, London A, Schwartz M. Orchestrated leukocyte recruitment to immune-privileged sites: absolute barriers versus educational gates. Nat Rev Immunol. 2013;13(3):206-18. doi: 10.1038/nri3391.

7. Nguyen HX, Hooshmand MJ, Saiwai H, Maddox J, Salehi A, Lakatos A, Nishi RA, Salazar D, Uchida N, Anderson AJ. Systemic Neutrophil Depletion Modulates the Migration and Fate of Transplanted Human Neural Stem Cells to Rescue Functional Repair. J Neurosci. 2017;37(38):9269-9287. doi: 10.1523/JNEUROSCI.2785-16.2017.

8. Shechter R, Miller O, Yovel G, Rosenzweig N, London A, Ruckh J, Kim KW, Klein E, Kalchenko V, Bendel $P$, Lira SA, Jung S, Schwartz M. Recruitment of beneficial M2 macrophages to injured spinal cord is orchestrated by remote brain choroid plexus. Immunity. 2013;38(3):555-69. doi:

10.1016/j.immuni.2013.02.012.

9. Wang J, Ma MW, Dhandapani KM, Brann DW. Regulatory role of NADPH oxidase 2 in the polarization dynamics and neurotoxicity of microglia/macrophages after traumatic brain injury. Free Radic Biol Med. 2017;113:119-131. doi: 10.1016/j.freeradbiomed.2017.09.017.

10. Shapouri-Moghaddam A, Mohammadian S, Vazini H, Taghadosi M, Esmaeili SA, Mardani F, Seifi B, Mohammadi A, Afshari JT, Sahebkar A. Macrophage plasticity, polarization, and function in health and disease. J Cell Physiol. 2018;233(9):6425-6440. doi: 10.1002/jcp.26429. Epub 2018 Mar 1.

11. Fan H, Zhang K, Shan L, Kuang F, Chen K, Zhu K, Ma H, Ju G, Wang YZ. Reactive astrocytes undergo M1 microglia/macrohpages-induced necroptosis in spinal cord injury. Mol Neurodegener. 2016;11:14. doi: 10.1186/s13024-016-0081-8.

12. Kigerl KA, Gensel JC, Ankeny DP, Alexander JK, Donnelly DJ, Popovich PG. Identification of two distinct macrophage subsets with divergent effects causing either neurotoxicity or regeneration in the injured mouse spinal cord. Version 2. J Neurosci. 2009;29(43):13435-44. doi: 10.1523/JNEUROSCI.3257-09.2009.

13. Jia Z, Zhu H, Li J, Wang X, Misra H, Li Y. Oxidative stress in spinal cord injury and antioxidant-based intervention. Spinal Cord. 2012;50(4):264-74. doi: 10.1038/sc.2011.111. Epub 2011 Oct 11.

14. Yamamoto N, Ishikuro R, Tanida M, Suzuki K, Ikeda-Matsuo Y, Sobue K. Insulin-signaling Pathway Regulates the Degradation of Amyloid $\beta$-protein via Astrocytes. Neuroscience. 2018;385:227-236. doi: 10.1016/j.neuroscience.2018.06.018.

15. Lee S, Kim DC, Baek HY, Lee KD, Kim YC, Oh H. Anti-neuroinflammatory effects of tryptanthrin from Polygonum tinctorium Lour. in lipopolysaccharide-stimulated BV2 microglial cells. Arch Pharm Res. 2018;41(4):419-430. doi: 10.1007/s12272-018-1020-8.

16. Okada S, Nakamura M, Katoh H, Miyao T, Shimazaki T, Ishii K, Yamane J, Yoshimura A, Iwamoto Y, Toyama Y, Okano H. Conditional ablation of Stat3 or Socs3 discloses a dual role for reactive 
astrocytes after spinal cord injury. Nat Med. 2006;12(7):829-34. doi: 10.1038/nm1425.

17. Chen X, Liu L, Qian R, Liu J, Yao Y, Jiang Z, Song X, Ren J, Zhang F. Expression of Sam68 Associates with Neuronal Apoptosis and Reactive Astrocytes After Spinal Cord Injury. Cell Mol Neurobiol. 2017;37(3):487-498. doi: 10.1007/s10571-016-0384-x.

18. Nathan FM, Li S. Environmental cues determine the fate of astrocytes after spinal cord injury. Neural Regen Res. 2017;12(12):1964-1970. doi: 10.4103/1673-5374.221144.

\section{Figures}



A
B
C
D

GAPDH

\title{
Arginase-1
}

\author{
IL-10
}

IL-1及

TNF- $\alpha$

\section{Figure 1}

Expression of macrophage markers at 7 days after $\mathrm{SCl}$ detected by western blot analysis. A:Sham group; B: Model group; C: mmu-miR-27a-5p group; D: mmu-miR-27a-5p-negative control group 


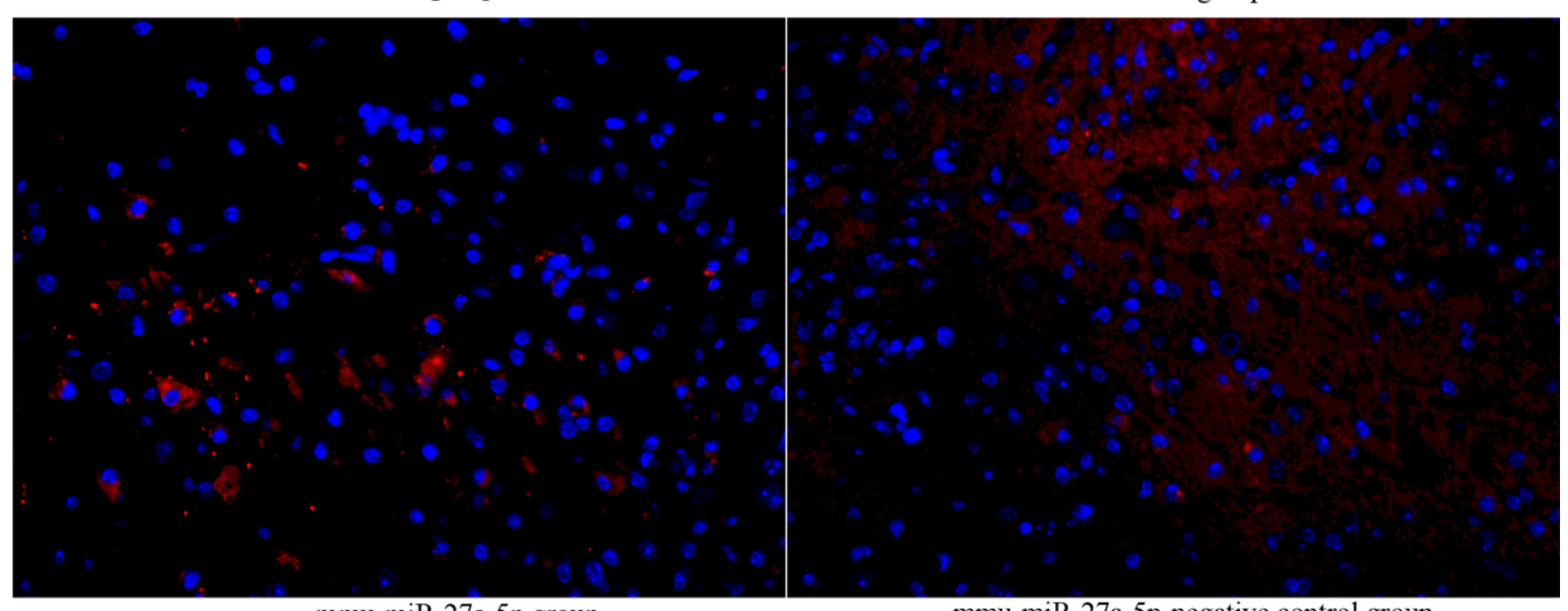

\section{Figure 2}

Immunofluorescent staining for macrophage polarization-associated markers at 7 days after SCl. Red fluorescence indicates the expression of IL-1 $\beta$ and TNF- $a$, green fluorescence indicates the expression of Arginase-1 and IL-10 

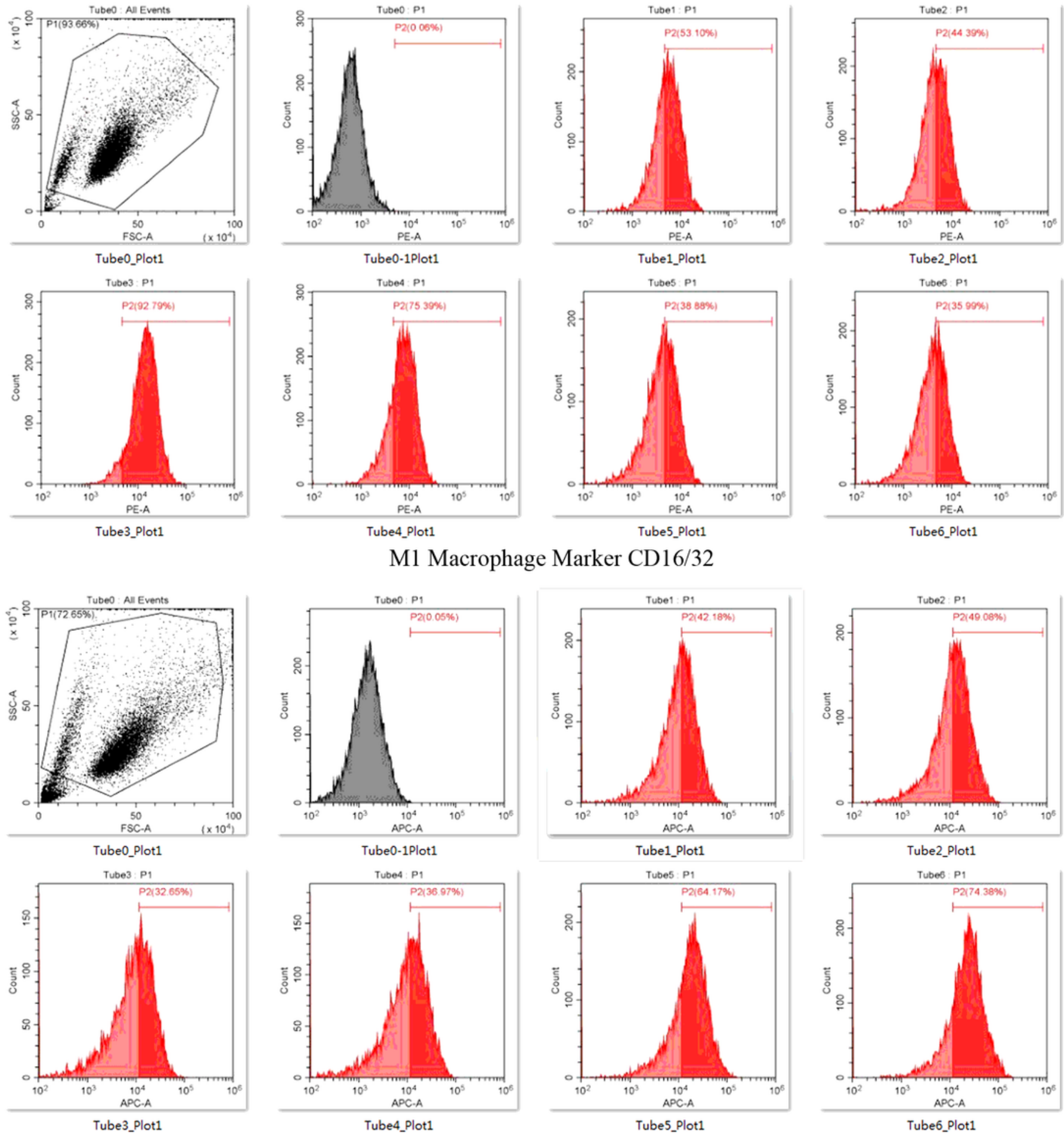

M2 Macrophage Marker Arginase 1

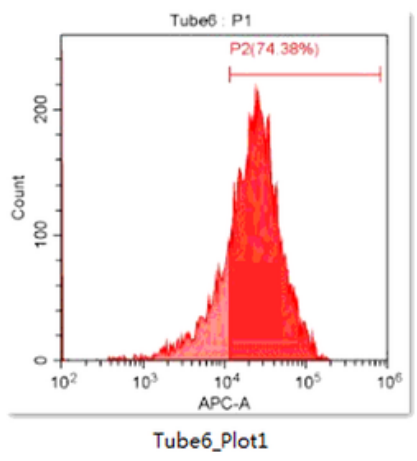

Figure 3

Flow cytometric analysis of different polarization types of macrophages 